\title{
Sociedades heterárquicas en el Ecuador preincaico: estudio diacrónico de la organización política caranqui
}

\author{
María Fernanda Ugalde y Cristóbal LandÁzURI NARVÁEZ \\ Pontificia Universidad Católica del Ecuador \\ mfugalde@puce.edu.ec clandazurin@puce.edu.ec
}

Recibido: $17 / 9 / 2015$

Aceptado: 7/7/2016

\begin{abstract}
RESUMEN
El área cultural caranqui, localizada en la Sierra Norte del Ecuador, comprende un conjunto homogéneo en términos de cultura material caracterizado principalmente por la presencia de complejos monumentales conformados por montículos artificiales, que en la terminología local se denominan tolas, y que tienen la forma de pirámides truncadas, ocasionalmente acompañadas de enormes rampas de acceso. La cultura material mueble asociada a estos monumentos, en particular la cerámica, es también bastante homogénea y cuenta con dos formas tipo, un ánfora grande y una olla asimétrica trípode. Este conjunto cultural data de la época preincaica tardía, y está fechado aproximadamente entre 1250 y 1525 d.C. Se ha tratado de explicar la organización política de estas sociedades desde modelos jerárquicos como el de la Teoría del Lugar Central, buscando una capital que hubiera ejercido poder y control sobre los otros sitios. Las fuentes etnohistóricas no sustentan una hipótesis en ese sentido, y hablan más bien a favor de señoríos relacionados pero autónomos. En este artículo proponemos una visión distinta de la organización política caranqui, desde el modelo de heterarquía, postulando que los sitios con montículos no fueron centros de poder político sino religioso. Sustentamos esta idea a través de un estudio diacrónico que combina las fuentes arqueológicas y las etnohistóricas.
\end{abstract}

Palabras clave: Ecuador, caranqui, heterarquía, complejidad social, organización política

\section{Heterarchical Societies in Pre-Inca Ecuador: A Diachronic Study of Caranqui Political Organization}

\begin{abstract}
The Caranqui cultural area, located in the Northern Highlands of Ecuador, comprises a homogeneous whole in terms of material culture, mainly characterized by monumental complexes formed by mounds (referred to as tolas in the local terminology), which tends to have the shape of a truncated pyramid, occasionally accompanied by huge access ramps. The material culture associated with these monuments, in particular ceramics, is also fairly homogeneous and has two type forms, a large amphora and an asymmetric tripod pot. This cultural complex is present from late pre-Inca times and is dated approximately between 1250 and 1525 A.D. There have been several attempts to understand the political organization of these societies through hierarchical models such as Central Place Theory, seeking a capital city that had exercised power and control over other sites. Ethno-historical sources do not support such a hypothesis, and rather, they seem to favor a series of related but independent chiefdoms or manors. In this article we propose a different view of Caranqui's political organization, through a heterarchy model, postulating that the sites with mounds were not political but rather religious power centers. We support this idea through a diachronic study combining archaeological and ethnohistorical sources.
\end{abstract}

Key words: Ecuador, Caranqui, heterarchy, social complexity, political organization.

Sumario: 1. Introducción. 2. Heterarquía. Un modelo alternativo para el análisis de estructuras de organización en la escala regional. 3. Los datos arqueológicos. 4. Los datos etnohistóricos. 5. Discusión. El estudio diacrónico y sus aportes a la investigación del área cultural caranqui. 6. Conclusiones. 7. Referencias bibliográficas. 


\section{Introducción}

La presente investigación se centra en la organización política del área cultural caranqui antes y después de las conquistas incaica y española. En esta zona de la Sierra norte del Ecuador tuvieron lugar drásticos cambios en un lapso relativamente corto, comprendido entre el período de Integración Tardío de la época preincaica, la incursión incaica y la conquista española. Las evidencias de estos cambios, tanto materiales como inmateriales, se encuentran básicamente en dos tipos de fuentes: las arqueológicas y las etnohistóricas.

A lo largo de la investigación científica relacionada con el área cultural caranqui, existe un consenso acerca de que se trató de sociedades complejas, que compartieron tanto una lengua como una cultura material evidenciada fundamentalmente a través de imponentes conjuntos de montículos artificiales. No obstante, no se ha podido especificar la forma en la que los distintos grupos que ocuparon la zona estuvieron relacionados entre sí. Las fuentes coloniales dejan constancia de la existencia de una lengua común (Borja 1965: 249), así como de la presencia de varios señoríos con poderosos dignatarios (Caillavet 2000; Larraín 1980; Moreno 2007). Desde la arqueología, el exhaustivo estudio de Athens (1980) aborda y confirma la complejidad en la organización social, centrando su análisis especialmente en la inversión de energía requerida para la construcción de los montículos artificiales, pero no ofrece una potencial explicación acerca de la modalidad a través de la cual estaban relacionados unos y otros asentamientos. Otros estudios (Athens 2010, 2012; Cordero 2009; $\mathrm{Cu}-$ rrie 2001; Oberem y Wurster 1989; entre otros) se han centrado en un sitio específico y los detalles de su posible organización social, dejando también fuera de su análisis el aspecto relativo a la relación intrarregional entre los diversos sitios.

Por tanto, considerando este aspecto un vacío en la investigación, pretendemos abordar aquí el estudio del área en cuestión desde esta perspectiva, pero alejándonos del modelo jerárquico normalmente implícito en los análisis de sociedades complejas a nivel regional.

\section{Heterarquía. Un modelo alternativo para el análisis de estructuras de organización en la escala regional}

La mayoría de los modelos que pretenden explicar las formas de relación entre grupos humanos en la escala regional se sirve de teorías que se asientan en estructuras jerárquicas, asumiéndose que la jerarquía sería la única forma posible en la que pueden estar organizadas las estructuras sociales (Crumley 1995: 2). No es la única. Una estructura heterárquica consiste en una organización cuya forma puede compararse a la de una red, a diferencia de la forma piramidal que caracteriza la organización jerárquica. Mientras la conexión entre elementos en una estructura jerárquica se suele representar verticalmente, en una estructura heterárquica la conexión es horizontal (Crumley 1979). La aplicación que pretendemos dar aquí a estos conceptos está relacionada con la organización política a nivel regional, girando la pregunta principal alrededor de la forma en la que el poder es ejercido dentro de la región estudiada y 
de qué tipo de poder se trata. Si entendemos a las sociedades como sistemas estructurados, una estructura jerárquica es aquella que cuenta con un poder centralizado que gobierna sobre un territorio, mientras que una estructura heterárquica podría entenderse como un conjunto de sociedades que comparten un territorio y una serie de elementos culturales (arquitectura, repertorio cerámico, idioma, etc.), pero entre las cuales no hay una que tenga autoridad y poder de decisión sobre las otras.

Esta perspectiva teórica ha sido abordada desde los años setenta en relación con el entendimiento de sociedades pretéritas (Crumley 1979; Ehrenreich et al. 1995; entre otros), pero en el Ecuador prácticamente no se ha tomado en cuenta para la interpretación en arqueología, y puede considerarse un paradigma casi desconocido (con la única excepción de Bray 2008). Existen varios ejemplos en la literatura especializada que plantean una organización heterárquica para sociedades complejas, como es el caso de los celtas (Crumley 1979), los mayas (Potter y King 1995), y los araucanos (Dillehay 2006), entre otros. Es necesario enfatizar que el modelo heterárquico no implica en absoluto que se trate de sociedades igualitarias; el modelo se refiere a la escala regional, y plantea una distribución no-centralizada del poder, pero que sin excluir la existencia de jerarquías en el interior de las unidades que componen el entramado, es decir, en nuestro caso de estudio, los diferentes grupos sociales asentados en el área caranqui.

Los datos arqueológicos demuestran claramente que, por ejemplo en Cochasquí - para citar el caso más ampliamente estudiado dentro del área de investigación- la sociedad estuvo organizada internamente en forma no-igualitaria, como se evidencia a través de los patrones de enterramiento observados. Existen, de un lado, entierros ricos, con ofrendas finas y numerosas, y en los que se invirtió una considerable cantidad de energía para la construcción de las tumbas (Wentscher 1989), y de otro lado, entierros muy simples, con pocas o ninguna ofrenda funeraria (Ugalde 2015; Wentscher 1989).

Estudios de caso como el de Potter y King (1995), relativo al área maya, presentan evidencias de la convivencia de jerarquía y heterarquía en la misma sociedad, pero en diferentes escalas, entendiéndose con ello el hecho de que los grupos sociales están determinados en su interior por estructuras jerárquicas, cuyas élites buscan la forma de justificar y perpetuar su situación privilegiada, pero en la escala regional se observa que estas élites no jugaron ningún papel dominante en la economía ni tuvieron una organización centralizada de poder y administración. Una organización similar en el área cultural caranqui constituye el supuesto guía de la presente investigación.

\section{Los datos arqueológicos}

La presente investigación aborda el estudio de lo que en la literatura especializada se conoce como área cultural caranqui, y que corresponde a una sociedad compleja asentada en una porción de la Sierra norte del Ecuador, entre los ríos Chota-Mira por el norte y Guayllabamba por el sur, habiendo ocupado un área de alrededor de 3600 $\mathrm{km}^{2}$ (Bray 2003: 17, 2008: 528). Temporalmente, el estudio del componente arqueológico se centra en el período de Integración, de acuerdo con la periodización tradi- 
cional $^{1}$, o en el período Tardío, según la denominación de Athens (1980), es decir, la ocupación directamente anterior a la incursión incaica en la zona.

El área está claramente caracterizada por la presencia de complejos monumentales constituidos por montículos artificiales de diferentes formas, conocidos localmente como tolas. La mayoría de ellos tienen forma de pirámide truncada, y algunos fueron dotados de rampas de acceso extremadamente largas. Existen también montículos de forma hemisférica, los cuales son mucho menores en tamaño que los piramidales y parecen estar asociados en su mayoría con funciones funerarias. Dada su gran visibilidad en el paisaje, estos bienes culturales son conocidos desde épocas tempranas y han sido registrados e inventariados en algunas ocasiones (Athens 1980, 2003; Gondard y López 1983), pero aún son muy pocos los que han sido excavados arqueológicamente.

Jijón y Caamaño fue el primero que trató de organizar los hallazgos del área cultural caranqui bajo el criterio de su arquitectura monumental, proponiendo una diferenciación entre lo que denominó «Civilización de las tolas con pozo» y la «Civilización de las tolas habitacionales», dejando ya establecida la distinción tanto morfológica como funcional entre los montículos hemisféricos y los montículos cuadrilaterales con o sin rampa. Según este autor, las «tolas con pozo» serían de carácter funerario, y menciona como ejemplo algunas excavados por él en Urcuquí (Jijón y Caamaño 1997: 312). En cambio los montículos cuadrilaterales serían de carácter habitacional, y entre ellos menciona a los conjuntos de Cayambe, Atuntaqui, Zuleta y Cochasquí (1997: 314). Es en este último sitio donde se han realizado las investigaciones arqueológicas más exhaustivas y que nos proporcionan una amplia gama de informaciones acerca de varios aspectos, entre los que podemos resaltar las técnicas constructivas de los montículos, indagaciones sobre las diferencias funcionales entre montículos piramidales y montículos hemisféricos, datos de cronología relativa y absoluta, tipologías de cerámica y lítica, análisis de restos óseos, etc. Cochasquí cuenta con 15 montículos piramidales, 9 de ellos con rampa, además de 11 montículos hemisféricos, y ha sido objeto de diversos estudios basados en trabajos arqueológicos in situ (Oberem 1981, ed.; Oberem y Wurster 1989; Ugalde 2015; Uhle 1933, 1939).

La clasificación cerámica efectuada por Meyers sobre el material de este sitio (Meyers 1981, 1989) se toma por válida en términos generales para toda el área. En ese trabajo, Meyers estableció dos fases cronológicamente sucesivas, Cochasquí I (950 y 1250 d.C.) y Cochasquí II ( 1250 y 1550 d.C. $)^{2}$. La forma tipo del conjunto temprano es la olla zapatiforme, una tipo de vasija cerrada asimétrica, mientras que las formas más características del conjunto tardío serían las vasijas trípodes y las ánforas. Las decoraciones en la cerámica local son muy escasas y en general el corpus cerámico es de carácter más bien tosco.

\footnotetext{
1 El período de Integración se caracteriza principalmente por la intensificación y tecnificación de la agricultura y el surgimiento de grandes áreas culturales (centros urbanos) donde tiene lugar una especialización en las tareas laborales y una diferenciación de clases sociales (Meggers 1966: 119). En términos cronológicos, se ubica esquemáticamente entre el año 500 d.C. y la llegada de los incas.

2 Fases propuestas después del contraste de dataciones radiocarbónicas, contextualizadas estratigráficamente, y formas cerámicas (Meyers 1981, 1989; Narr y Schönfelder 1989).
} 
En lo que concierne a la lítica, son sumamente escasos los análisis especializados, y es nuevamente la investigación de materiales de Cochasquí la que nos proporciona el mayor detalle en cuanto al análisis. El material lítico de Cochasquí se compone principalmente de herramientas elaboradas en obsidiana y basalto. Mientras el basalto probablemente fue extraído del volcán Mojanda, en las inmediaciones mismas de Cochasquí, la obsidiana proviene de una fuente lejana al sitio (Zalles-Flossbach 1989). Del análisis de fluorescencia de rayos X recientemente realizado (Dyrdahl 2015) se desprende que la obsidiana proviene primordialmente de las fuentes de $\mathrm{Mu}-$ llumica y Callejones. La importancia de esta observación se resaltará y discutirá en el siguiente acápite.

Otros sitios investigados que vale la pena mencionar son Zuleta, que con 148 montículos en total es el mayor conjunto arquitectónico de toda el área de estudio (Athens 1980, 2010, 2012; Currie 2001), Puntiachil (Buys et al. 1991; Cordero 2009), Socapamba, Pinsaquí y Gualimán (Athens 1980). De las excavaciones de Athens en Socapamba y Pinsaquí se obtuvieron dataciones radiocarbónicas que ubican la construcción y ocupación de los montículos artificiales entre 1250 y 1525 d.C., momento equivalente a la ocupación Cochasquí II que se encuentra bien documentada a través de un buen número de fechas radiocarbónicas (Narr y Schönfelder 1989).

Nuestra investigación incluyó una revisión exhaustiva no solo de las publicaciones relativas al área cultural caranqui, sino de cientos de informes inéditos, en su mayoría de arqueología de rescate, que yacen en el Instituto Nacional de Patrimonio Cultural (ver Ugalde 2015). Luego de la sistematización de esa información, observamos que 26 sitios arqueológicos del área cuentan con montículos con rampa, y casi todos ellos tienen también montículos sin rampa, en un número significativamente mayor a los primeros. Tras esta revisión, queda patente el hecho de que existen una serie de sitios con montículos con rampa de características similares en el área cultural caranqui. Como ha señalado Athens, este hecho habla a favor de que hubo varios centros de poder, y «No hay nada sugestivo de 'jerarquías funcionales' entre los sitios, que pudiera indicar una unificación política de la región» (Athens 1980: 188).

La influencia de la ocupación incaica en el área de estudio es notoria y se manifiesta tanto en las fuentes arqueológicas como en las etnohistóricas. Algunos sitios aún conservan arquitectura asociada a la presencia inca, como la imponente estructura de posible función ceremonial que se encuentra en Caranqui, en plena ciudad de Ibarra (Bray y Echeverría 2014), así como un imponente complejo de pukaras (Oberem et al. 1968; Plaza Schuller 1977) que parece evidenciar la importancia de la defensa incaica a lo largo de su avanzada en este territorio frente a una feroz resistencia caranqui ${ }^{3}$.

\section{Los datos etnohistóricos}

Se han revisado especialmente las fuentes tempranas, partiendo del supuesto de que las informaciones que traen estos papeles son más cercanas a la realidad prehispánica

\footnotetext{
3 Aunque se ha planteado la posibilidad de que algunas de estas estructuras defensivas sean de origen preincaico, es indudable su utilización por parte de los incas en el marco de su expansión bélica hacia el norte.
} 
debido a que los procesos de conquista y de dominación del modelo colonial español aún no habían modificado sustancialmente a las sociedades nativas. Este tipo de fuentes son principalmente crónicas, relaciones y documentación administrativa como numeraciones, visitas, tasas tributarias, litigios de tierras y testamentos. En algunos casos se tuvo que recurrir a documentos del siglo XVII, cuando no existen otros más tempranos para las zonas de interés. El análisis se realizó a partir de tres indicadores: población, sistemas de producción y circulación y organización política. Se asume que el tamaño de la población está en relación directa con la complejidad de la organización política de unidades, y la evidencia encontrada refleja diversos tamaños de ayllus, parcialidades y cacicazgos contenidos en las unidades políticas, lo que parece hablar a favor de niveles de jerarquía y relaciones de poder y autoridad entre ellas. El indicador relativo a los sistemas de producción y circulación se encuentra en documentos como informes sobre la producción, litigios de tierras, sobre la agricultura nativa, la existencia de especialistas en comercio y prácticas de intercambio, entre otros. La reconstrucción de estos sistemas permite realizar inferencias acerca del manejo de los recursos, tierra y agua, desde los centros de poder; de igual manera identificar las formas de control de la mano de obra y su centralización. En el caso de los sistemas de circulación, la identificación de especialistas de intercambio (mindalaes) o centros de mercados, llevan a pensar en relaciones de redistribución que generalmente están asociados con agentes redistribuidores y relaciones jerárquicas, siguiendo la clásica interpretación andina sobre los caciques y sus fondos de poder sustentados en su capacidad redistributiva. En cuanto a la organización política, sin duda este indicador es el más débil de los tres, debido a la ausencia de información directa. Los datos que se manejan permiten únicamente inferencias indirectas, y se los encuentra en registros de numeraciones, en los roles que el Estado colonial asignó a las elites nativas, al cobro y monto de tributo que manejaban y administraban los caciques.

En el espacio investigado, la mayoría de estudios históricos concuerdan en que existieron tres grandes unidades políticas: Otavalo, Caranqui, y Cayambe (Cuadro 1).

El Cacicazgo de Otavalo es el mejor documentado; una tasa tributaria realizada en 1577 en la que se fijó la cantidad de algodón que debía recibir cada cacique para confeccionar las mantas como tributo, enumera catorce pueblos: Otavalo, Zarance, San Pablo, San Juan, Tontaquí, Urcuquí, Tambabiro, Las Salinas, Cotacachi, Ynta, Guayllabamba, Axangue, Puellaro y Alchipichi, y al mismo tiempo registró un total de treinta parcialidades (Caillavet 2000: 150). Tomando como referencia la magnitud de algodón repartido y la distribución de parcialidades por pueblo, se evidencian tres estructuras políticas menores contenidas dentro del gran cacicazgo de Otavalo: Sa-

Cuadro 1: Unidades políticas en el área cultural caranqui según las fuentes etnohistóricas

\begin{tabular}{ll}
\hline Cacicazgo de Otavalo & Saranze \\
& Urcuquí \\
& Tontaquí / Cotacache \\
Cacicazgo de Caranqui & Caranqui \\
& Pimampiro \\
Cacicazgo de Cayambe & Cayambe \\
& Cochasquí \\
\hline
\end{tabular}


ranze, Urcuquí/Salinas y Cotacachi/Tontaquí. Saranze constituyó el principal centro demográfico, e incluía los pueblos de San Pablo, Imbaquí y San Juan. Fue el centro político y allí residieron los Ango de Salazar, linaje al que pertenecieron los caciques principales durante el periodo colonial (Ango de Salazar 1648).

Para la región de Urcuquí, que actualmente incluye la ciudad de Urcuquí (las parroquias de San Blas, Tumbabiro, Cahuasquí, Pablo Arena, La Merced de Buenos Aires y Salinas), en la época temprana se describen cuatro asentamientos que probablemente corresponden a cuatro pueblos menores: Urcuquí, como centro político, Tumbabiro, Cahuasquí, Las Salinas y Quilca. Los cuatro primeros se ubican en los pisos templados en torno a los 2400 m s.n.m., entre los ríos Ambi, Santiago o Puchinbuela y Palacara. En cambio Quilca está en la cordillera occidental, sobre los 3000 m s.n.m. (Paz Ponce de León 1582).

En la región de Tontaquí y Cotacache la tasa de 1577 menciona a los dos pueblos, cada uno con cuatro parcialidades, sin duda el primero con mayor tamaño demográfico y probablemente de mayor presencia política. Cotacache parece que tuvo como parcialidad anexa el pueblo de Ynta en la ceja de montaña occidental.

El cacicazgo de Caranqui estuvo localizado en la sección nororiental del territorio. De los datos disponibles se puede inferir que su territorio se extendió desde el valle del Chota hasta la zona de Angochagua-Zuleta, en el oriente del cerro Imbabura. En el valle del río Taguando o Caranquise se localizó lo que fue la cabeza del cacicazgo, el pueblo de Caranqui, al sur de lo que es hoy la ciudad de Ibarra. Manejando diversas fuentes es posible distinguir dos unidades políticas: Carangue, probablemente con tres parcialidades Cochicarangue, Carangue y San Antonio de Carangue. El centro político de este cacicazgo parece que fue Carangue, lo que se evidencia en la construcción inca denominada Aposentos de Carangue que, según Betanzos, fue construido por Atahualpa en una de sus expediciones hacia los pueblos pastos (Betanzos 1987: 217-220). Sobre Cochicarangue se menciona su reducción en el pueblo de Carangue, que bien puede ser lo que actualmente corresponde al sector de la Rinconada y de Angochagua.

El distrito de Pimampiro, como se lo ha delimitado anteriormente ubicado en la cabecera de la cuenca del río Chota, abarcó diferentes asentamientos; así, en su sección occidental se agruparon en lo que se designaba como el valle de Coangue, y existieron cuatro pueblos o parcialidades que fueron reducidos en Ambuqui (Borja 1965). En cambio, en la zona alta al oriente de Pimampiro está Chapi, actual pueblo de Shanshipamba (Bray 2001) que tempranamente fue reducido en el propio pueblo de Pimampiro.

El cacicazgo de Cayambe está localizado en la región más meridional del territorio caranqui. Esta área estuvo integrada por dos espacios ecológicamente distintos y contiguos: la zona alta de Pesillo, Cayambe, Tabacundo, Cochasquí, Malchinguí y Alance; y la zona de baja y cálida del valle del río Guyallabamba, con los pueblos de Puéllaro, Perucho, Lalchipi, Iranquí y Guayllabamba. Cayambe aparentemente que fue el centro político e incluyó a tres pueblos de Puntoyachil, (el actual Cayambe) Tabacundo, la Chimba en el lado oriental de Imbabura, y Guayllabamba o Iraquí, que parece que fue un enclave de producción especializada políticamente ligado al cacicazgo de Cayambe (Moreno 1982; Ramón 1987). El rango político de Cayambe está 
documentado en la información de servicios que hizo Hierónimo Puento en 1583, en donde testifica su participación en las guerras de conquista y pacificación de la región de Quijos, en las primeras décadas del período colonial (Puento 1974).

El otro pueblo de este cacicazgo fue Cochasquí. Su mención es igualmente temprana en una petición hecha en 1564 por algunos caciques, en la que aparece firmando el documento Alonso Andaparinango cacique del pueblo de Cochisqui (Vargas 1977: 123). En la jurisdicción de esta zona se incluyen dos pueblos, Puéllaro y Perucho en la cuenca del rio Guayllabamba. Por su localización parece que constituyeron un espacio de producción especializada de manera especial de coca, en donde también estuvieron presentes los señores de Otavalo.

\section{Discusión. El estudio diacrónico y sus aportes a la investigación del área cultural caranqui}

Una vez expuestos los datos conocidos sobre el material cultural del área cultural caranqui en la época prehispánica tardía, debemos preguntarnos ¿en qué medida la cultura material, tanto mueble como inmueble, podría constituirse como un indicador sobre el tipo de organización política, y evidenciar si esta fue jerárquica o heterárquica en la escala regional?, y ¿cómo se articulan estos datos de cultura material con aquellos que nos proporcionan las fuentes escritas? El aspecto económico parece ser crucial aquí, ya que la producción centralizada y de gran escala es uno de los indicadores principales dentro de un modelo de organización regional jerárquica (Bray 2003). El estudio de Potter y King (1995) en el área maya sugiere que, en cambio, en sociedades complejas con organización de tipo heterárquico, la producción especializada no es centralizada ni tiene lugar bajo el control de una élite gobernante a nivel regional, sino que existen especialistas a nivel local que abastecen a sus comunidades con los productos necesarios. Igualmente en el ámbito comercial, estos autores proponen que en sociedades con organización heterárquica, el comercio no está controlado en forma centralizada, sino que se maneja individualmente a partir de cada unidad autónoma. Vamos a analizar a continuación, con ayuda de los indicadores de los que disponemos, estas interrogantes.

Para la cerámica, en general es difícil identificar en el registro arqueológico restos tangibles relacionados con su manufactura. Un indicador accesible, sin embargo, es la composición de las pastas en contraste con los depósitos de arcilla y su localización (Potter y King 1995: 25). En este sentido, si bien no contamos en nuestra área de estudio con restos directos sobre la producción de cerámica, como serían hornos relacionados con su fabricación, la materia prima utilizada para la confección de la alfarería caranqui indica que su producción tuvo lugar a nivel de comunidades y no centralizada, con arcillas residuales desarrolladas in situ a partir de material volcánico (Bray 2003: 71). Estos datos contrastan con aquellos presentados por Bray (2003: 13) para la época de la ocupación inca en la zona, donde pudo observar algunas diferencias, aunque menores, en el grado de elaboración técnica y en la estandarización morfológica de la cerámica, que interpretó como un aumento en el control administrativo sobre la economía local. 
En cuanto al aspecto morfológico de la cerámica, existe una forma particular que requiere mención y reflexión, el ánfora. Se trata de una vasija grande, cerrada, con base en forma de punta y frecuentemente decorada con pintura roja en bandas, a la que Meyers denominó «ánfora Cochasquí». Este tipo de vasija es, junto con la olla trípode, la forma tipo de la fase Cochasquí II (Meyers 1989). Mientras las ollas trípodes son omnipresentes en el área cultural caranqui, independientemente de los contextos, las ánforas parecen restringirse a los contextos de montículos con rampas. Su presencia está evidenciada para las áreas monticulares de Cochasquí (Meyers 1989), Puntiachil (Cordero 2009), Socapamba, Cahuasquí (Athens 1980) y Zuleta (Athens 2010). Mientras tanto, en espacios no-monumentales cercanos a los montículos, este tipo de vasijas parecen estar ausentes. Esta observación constituye un indicio acerca de la función de este tipo de vasijas que, con bastante fiabilidad, puede relacionarse con el almacenamiento de bebidas, específicamente con la chicha, y estaría vinculada con la realización de celebraciones masivas (Athens 2010: 3; Bray 2003: 143-144).

Creemos además que la presencia de estas ánforas, aparentemente en todos los sitios de montículos con rampa, puede interpretarse en el sentido de que todos ellos constituyeron centros de poder con énfasis ceremonial. De haber existido un sitio que ostentara el poder centralizado, debería éste contar con la exclusividad de este tipo de vasijas. Este elemento podría entonces constituir un primer indicador a favor de una organización heterárquica en el área cultural caranqui.

Otras interesantes observaciones sobre la cerámica y sus implicaciones socioeconómicas se desprenden del estudio efectuado por Bray (2003), encaminado a entender el impacto de la intervención incaica en el área cultural caranqui. Aquí, Bray se cuestiona si tuvieron lugar grandes cambios como resultado del mayor control político (por parte de una sociedad estatal como la incaica) sobre la región caranqui, y plantea que las evidencias de tales cambios deberían manifestarse en la producción artesanal (parte importante de la cual fue la producción de cerámica), concluyendo que ciertas diferencias en cuanto a elaboración, técnica y estructura morfológica de la cerámica de sitios inca, apuntan a algún incremento en el grado de estandarización (Bray 2003: 13). Esta evidencia de control centralizado de la producción artesanal se entiende en contraste con la ausencia del mismo en la época previa a la ocupación inca del área de estudio.

Además de la producción, es importante analizar el aspecto de las relaciones comerciales y su posible naturaleza. Sobre esto, es interesante el hecho de que en varios de los sitios con montículos con rampa estudiados se ha registrado, a la par de la cerámica de manufactura local, una cerámica importada que se conoce como Panzaleo o Cosanga. Se trata de una alfarería muy fina, de paredes sumamente delgadas y caracterizada por el brillo que le otorga el uso de la mica como desgrasante, y cuyo origen (a juzgar por la composición de la arcilla con la que fue confeccionada) se encuentra en las estribaciones occidentales de la cordillera oriental (Bray 1995; Fritz y Schönfelder 1986). Este tipo de alfarería fue registrada en contextos monticulares en Cochasquí (Oberem y Wurster 19894), Puntiachil (Cordero 2009) y Zuleta (Athens 2012), mientras que está prácticamente ausente en contextos no-monticulares, al me-

4 Ver aquí el capítulo de Schönfelder (1989) con el análisis cerámico de este componente. 
nos en lo poco que se ha investigado en estas zonas (Ugalde 2015). Esto hace pensar en un uso de esta cerámica exótica como producto de estatus por parte de las élites locales, pero, nuevamente, su presencia en varios sitios habla a favor de una organización sin poder centralizado, con poderes locales autónomos, todos los cuales tuvieron acceso a estos bienes de prestigio y los utilizaron probablemente tanto en sus prácticas cotidianas como en sus ajuares funerarios ${ }^{5}$.

La lítica, por otro lado, nos proporciona igualmente indicios para sustentar nuestra hipótesis. En particular nos sirven aquí los datos proporcionados por los análisis químicos que indican las posibles fuentes de obtención de la obsidiana, con los que contamos gracias al trabajo de Dyrdahl y Speakman (2013), en el que proponen que cada uno de los sitios que conformaron el área cultural caranqui tuvo un medio diferente para obtener obsidiana, lo cual habla claramente en contra de un control centralizado del sistema de comercio, al menos el relacionado con este material. El estudio comprendió el análisis de colecciones de obsidiana de los sitios Huataviro (Ibarra) y Puntiachil (Cayambe), observándose un abastecimiento independiente y de distintas fuentes. Un análisis posterior de Dyrdahl (2015), realizado en la colección de Cochasquí obtenida por Ugalde en su prospección, es consistente con esos datos, conduciendo a Dyrdahl a proponer que el patrón de obtención más probable es que cada grupo era responsable de recolectar la obsidiana que consumía. Esto habla en contra de una sociedad organizada jerárquicamente a nivel regional y con un control político centralizado, pues en palabras de Dyrdahl (2015: 128): «para que existieran las redes desarrolladas de adquisición de obsidiana, esperaríamos que existiera un nivel más alto de demanda de materia prima».

En cuanto a la producción y el comercio, estos ámbitos resultan discernibles en buena medida a través de los documentos escritos e igualmente se pueden encontrar parcialmente sus huellas materiales a través de hallazgos arqueológicos. Este ámbito es a su vez fundamental para nuestra pregunta de investigación, ya que la organización política de una sociedad está estrechamente ligada con el sistema económico. Sabemos que la alimentación de la sociedad caranqui estuvo fundamentada en la práctica de la agricultura a gran escala, como evidencian una serie de hallazgos arqueológicos entre los que cuentan campos elevados (camellones), terrazas agrícolas, canales de riego y albarradas. Existen ejemplos de estos hallazgos, en parte conservados aún en nuestros días a gran escala, en Cayambe, Ayora, Perucho y cerca de Zuleta (Athens 1980, 2010; Cordero 2009; Gondard y López 1983; Villalba 2009). También las fuentes documentales mencionan la existencia de formas de cultivo que requirieron la modificación del paisaje:

«.... y el maíz en camellones, habiendo poco más de un pie de uno a otro, hácese un agujero con el dedo, échase dos granos de maíz y uno de los frisoles, cuando los quieren sembrar, los cuales como van cresciendo, se ciñen y abrazan con las cañas de maíz y ansí tienen fuerza para se levantar del suelo.... Siempre tienen sus labranzas muy deherbadas y limpias y mollentada la tierra ques causa de producir más y mejor.

5 En Cochasquí, los ajuares funerarios registrados en los montículos artificiales hemisféricos están frecuentemente compuestos por una combinación de vasijas de manufactura local y vasijas Cosanga (Wentscher 1989). 
Si ambrese el maíz de manera que está una macolla de otra como un pie» (Anónimo 1573: 189).

Como ha resaltado Athens, las evidencias conocidas hasta el momento hablan a favor de un importante desarrollo de sistemas para el aumento de la producción agrícola, que fueron adaptados estratégicamente en función de las condiciones locales, como el uso de sistemas de irrigación artificial en las áreas secas, el uso de campos de camellones en áreas pantanosas y la construcción de terrazas en las laderas escarpadas (Athens 1980: 123). No obstante no hay evidencias de un control centralizado de la producción agrícola, que en la arqueología se manifestarían principalmente a través de sistemas de almacenamiento de productos. Cuando existen este tipo de sistemas, sus manifestaciones materiales suelen presentarse entre el material arqueológico, como es el caso de los depósitos incaicos denominados colcas, donde el grado de especialización fue tal que estas estructuras incluso tuvieron formas diferentes según el producto que albergaban. Según Morris y Thompson, las colcas circulares se utilizaban tradicionalmente para almacenar maíz y las cuadrangulares para papas y otros tubérculos. Estos datos se desprenden de las excavaciones de estos autores en el centro administrativo de Huánuco Pampa, donde se conocen más de dos mil estructuras de almacenamiento (Morris y Thompson 1970: 356). Existe además un amplio registro arqueológico de este tipo de estructuras a lo largo del Tahuantinsuyu (Moseley 1992: 66; Rostworowsky 1988: 282).

En relación con este aspecto en el área cultural caranqui, las observaciones de Athens, basadas en sus excavaciones en Socapamba, coinciden con lo que aquí se está sosteniendo:

«No hay evidencia de almacenamiento de ningún tipo, como lo indican las excavaciones que ya hemos discutido, y la prospección de superficie. La carencia de una distribución diferencial de algún tipo de artefactos en Socapamba, también argumenta contra la presencia de productos especializados. Los argumentos que van contra la facilidad de almacenamiento también reciben apoyo de los documentos etnohistóricos y las observaciones etnográficas modernas del área de Otavalo. No hay datos etnohistóricos que establezcan la presencia de productores especializados (en los términos concebidos aquí) o de facilidades para almacenaje» (Athens 1980: 177).

Lo que sí indica la evidencia arqueológica es que algunos de los sitios con montículos se hallan asociados con los restos de sistemas agrícolas, entre ellos Puntiachil y Zuleta. Un patrón similar observado en Belice para el período Clásico Tardío maya ha sido interpretado como evidencia de un control autónomo de la producción agrícola y, por tanto, como parte de una organización política heterárquica (Hageman y Lohse 2003), ya que habla en contra del control centralizado de la producción y su almacenamiento y redistribución por parte de un grupo de poder, como sería el caso en una sociedad organizada jerárquicamente. Estos autores (2003: 121) identificaron en su área de estudio cuatro sitios ocupados por lo que denominan grupos corporativos, concluyendo que cada grupo fue responsable de su propia esfera de producción y que las relaciones que mantuvieron entre ellos fueron horizontales.

El desarrollo de la agricultura intensiva, base de la economía de los cacicazgos caranqui, estuvo sustentada en un conocimiento amplio del medio ambiente. Este conocimiento permitió a las sociedades desarrollar un sistema de agricultura multicíclica 
en la producción de los bienes de subsistencia básicos, lo cual se dio en los diversos pisos altitudinales con un aprovechamiento óptimo de la fuerza de trabajo, debido a que paralelamente se manejaban varios ciclos productivos. La papa, y en general los tubérculos, son cultivos de altura, con mayor resistencia a las heladas, cuyo rendimiento y adaptación encuentran sus mejores niveles en las zonas húmedas. El maíz, preferentemente de clima templado y presente en toda la región de estudio, requiere de mayor temperatura y su cultivo es más seguro en lugares con menos frecuencia de heladas. Atendiendo a la ubicación de los asentamientos caranqui registrados por las fuentes documentales, la gran mayoría y los más grandes numéricamente estuvieron ubicados en los pisos templados en torno a los $2500 \mathrm{~m} \mathrm{s.n.m.,} \mathrm{un} \mathrm{hábitat} \mathrm{ideal} \mathrm{para}$ la producción maicera. El cultivo de estos dos productos necesariamente significó dos sistemas o prácticas culturales que implicaron un conocimiento y una tecnología diversa de acuerdo con el ciclo agrícola, a las condiciones agrológicas del suelo y a la disponibilidad de agua y humedad. A pesar de considerarse como dos sistemas agrícolas, creemos que conformaban un mismo proceso de producción, limitado por las condiciones ecológicas, al uso y destino de los productos en función de satisfacer las necesidades alimenticias y de reproducción del grupo (sensu Murra 1975: 56, 57). De las fuentes escritas se desprende que el maíz fue el producto con mayor importancia dada su mayor capacidad de conservación, y seguramente por sus altos niveles de productividad debidos a las condiciones ambientales. Esto lleva pensar en que la población tuvo acceso directo a su producción y consumo y a la ausencia de centros de almacenamiento y de redes de redistribución a nivel intra-sitio.

El otro tema importante relativo a la producción atañe a las relaciones de trabajo dentro de una sociedad. A este respecto, las fuentes arqueológicas no presentan mucha evidencia, mientras que en las fuentes etnohistóricas se encuentra algunas referencias importantes. De los documentos tempranos se infieren varias modalidades de contratación de mano de obra vigentes en la época prehispánica, a saber: trabajo basado en relaciones recíprocas, mano de obra como tributo, contratación de indios foráneos, y mano de obra esclava como fue el caso de los yanas. Esta multiplicidad de mecanismos de acceso a la mano de obra corrobora la hipótesis de que los Señores étnicos contaban con mano de obra local y no se requería de redes centralizadas de acceso a la fuerza de trabajo.

En cuanto al comercio, tanto las fuentes arqueológicas como las etnohistóricas, nos proveen de importantes datos que permiten en alguna medida reconstruir los lazos comerciales y sus modalidades. La información arqueológica disponible apunta un sistema de intercambio no especializado, lo cual constituye un claro indicio en contra de una organización centralizada, y a favor de la hipótesis de este estudio. Como se señaló anteriormente, un importante indicador en este sentido es la obtención de la materia prima para la elaboración de herramientas líticas, en concreto la obsidiana. En trabajos recientes, Dyrdahl y Speakman (2013) y Dyrdahl (2015) concluyeron que no hay evidencias de un sistema unificado ni centralizado para la obtención de la obsidiana, y que más bien los análisis químicos indican que cada grupo obtuvo la obsidiana independientemente. En lo concerniente a la obtención de algunos productos exóticos como el algodón, Bray ha planteado que los mecanismos de obtención debieron operar a nivel doméstico (Bray 2003: 26). En lo que respecta a la cerámica 
Panzaleo o Cosanga, originaria de la región de la ceja de selva de la Cordillera Oriental (Bray 1995; Fritz y Schönfelder 1986), esta podría haber sido comerciada directamente por sus productores, a juzgar por su amplia distribución en la sierra norte y central del Ecuador, o bien a través del sistema de comunidades de paso, como ha sido propuesto para la hoya del Guayllabamba (Ugalde 2007).

Por su parte, los documentos históricos aportan significativas informaciones sobre los productos comerciados y las modalidades bajo las que tuvo lugar el intercambio. El análisis de estos documentos relativos a los Andes ha llevado a la producción de modelos que explican algunas particularidades en el sistema andino de producción y comercio. Partiendo del principio inferido por Murra (1975) sobre el máximo control de pisos ecológicos en los Andes Centrales, que le permitió desarrollar su modelo conocido como «Archipiélago vertical», Oberem desarrolló otro para los Andes Septentrionales. El modelo de economía microvertical (Oberem 1981) supone la ocupación de diversos pisos altitudinales por parte de una misma organización política, con fines de abastecimiento de los distintos bienes requeridos culturalmente. Entre ambos modelos (archipiélago vertical y microverticalidad) existe una diferencia fundamental de índole política y poblacional; mientras en el modelo micro hablamos de sociedades menos numerosas y con sistemas políticos menos complejos y menos centralizados, en el modelo extendido los pueblos son más numerosos y con sistemas políticos centralizados.

El comercio en el caso del área cultural caranqui responde claramente a un modelo microvertical y consecuentemente es factible hablar de un sistema heterárquico, en donde la contigüidad de los pisos altitudinales no requirió de estructuras organizativas complejas. Entre los caranquis, los pisos productivos de tubérculos y de maíz estuvieron contiguos y no requerían desplazamientos importantes. Su ocupación tuvo lugar probablemente a través de dos modalidades: directamente y por medio de $\mathrm{ka}$ mayukuna, es decir especialistas destinados temporalmente a esa tarea. La ocupación directa fue la utilización y producción de los pisos ecológicos en donde estaba asentado el grupo. La evidencia etnohistórica dice que los pueblos ocuparon generalmente los lugares de producción de maíz y papa, es decir los pisos desde los 2200 hasta los $3200 \mathrm{~m}$ s.n.m..

La ocupación y producción a través de kamayukuna consistió en la explotación de recursos en pisos contiguos al núcleo o centro de vivienda, por medio de unidades familiares desplazadas temporalmente a ese trabajo y que seguían manteniendo sus deberes y derechos en el núcleo; actividad que realizaban a nombre de su Señor étnico y eran parte de las relaciones de tributación entre Señores y sujetos. En algunos casos estos espacios tenían un carácter multiétnico, por cuanto eran zonas de producción de bienes suntuarios y exóticos como la coca, el algodón, la sal, y el ají entre otros. En territorio caranqui hay dos zonas claramente identificadas con estas características; el valle del Chota y el valle del Guayllabamba. Lo particular de este tipo de modelo es que las distancias entre núcleo y enclaves eran cortas, es decir que podían encontrarse a unas horas o hasta máximo un día de camino y estaban localizadas en los valles o en las cejas de montaña de occidente u oriente. Compatibles con este modelo, y empíricamente ejemplificadas, son las actividades de intercambio libre y dirigido, es decir, intercambio del tipo «comercio primitivo» entre unidades domésticas, y actividades 
de intercambio realizado por especialistas, los mindalaes. Entre los caranquis es ilustrativo el caso del cacicazgo de Cayambe, que ocupó las zonas de páramo sobre los $3200 \mathrm{~m}$ s.n.m. hasta zonas calientes en la cuenca del Guayllabamba en torno a los 2000 m s.n.m. (Ramón 1987); y el caso del cacicazgo de Caranqui que se extendió desde las zonas frías de Cochicaranqui hasta las playas del río Conague en torno a los 2000 m s.n.m. (Landázuri 1995).

\section{Conclusiones}

El concepto de heterarquía no es contradictorio con el de sociedades complejas. La complejidad social del área cultural caranqui ha sido objeto de estudio en más de una ocasión, y se ha demostrado detalladamente, además de haberse justificado con los respectivos sustentos teóricos (Athens 1980; Cordero 2009). El material cultural hallado en los pocos sitios estudiados arqueológicamente habla, efectivamente, a favor de una organización social jerárquica a nivel intra-sitio, hecho que no vamos a refutar en este estudio y que además encuentra su sustento en los documentos escritos tempranos. Nuestro interés aquí radica en la escala regional de análisis a nivel inter-sitio. Es aquí donde pensamos que el modelo de heterarquía es mucho más apropiado que otros modelos basados en la organización centralizada. Frecuentemente nos encontramos con estudios relativos al área cultural caranqui donde se busca encontrar un centro de poder político al estilo de una «capital», de alguna manera reproduciendo la organización política moderna. Aunque se reconoce la existencia de varios cacicazgos en la zona, se siguen buscando paradigmas que permitan encontrar cuál de estos fue el que mayor poder ostentó y relacionarlo jerárquicamente con respecto al resto de cacicazgos ${ }^{6}$. En esta discusión, los sitios con montículos con rampa del área cultural caranqui han sido considerados como los centros políticos, analizados mediante modelos como el de «vecino más próximo» (Athens 1980: 145) o la «Teoría del Lugar Central» (Brito et al. 2014). Sin embargo, los mismos autores mencionan un problema en este tipo de interpretación en virtud de que existen sitios grandes con montículos (pero sin montículos con rampa) que quedarían excluidos de la posibilidad de haber sido centros políticos como Perugachi, Gualsaquí y Angochagua (Brito et al. 2014). En nuestra opinión, y en virtud de las hipótesis esbozadas y los datos presentados a lo largo de este trabajo, consideramos que más bien los sitios monumentales pudieron corresponder a centros de poder religioso. Con este tipo de interpretación, se explican de mejor manera algunos aspectos que no han podido ser esclarecidos hasta hoy en las discusiones sobre la sociedad caranqui: (1) la relativa inconsistencia entre las fuentes arqueológicas y etnohistóricas, (2) la función de las pirámides, y (3) el carácter de la relación entre estos complejos. Abordaremos a continuación estos aspectos, a manera de síntesis interpretativa.

\footnotetext{
6 Ver discusión al respecto en Bray (2003: 19-20), que resume la divergencia de opiniones entre los etnohistoriadores Espinosa Soriano y Larraín. El primero sostiene que el cacicazgo caranqui fue el más poderoso, mientras que Larraín favorece al cacicazgo cayambe. Ambas propuestas se basan en las fuentes etnohistóricas $\mathrm{y}$ tienen poco sustento en las fuentes arqueológicas.
} 
Hasta el momento se cuenta con información de al menos 114 sitios arqueológicos con arquitectura monumental, de los cuales 26 tienen montículos con rampa y sin rampa, y 88 solamente con montículos sin rampa. Todos los montículos, con o sin rampa, cuando han sido excavados han demostrado tener en sus plataformas pisos de barro cocido con canales o artesas, que probablemente fueron hornos (Athens 1980: 149; Wurster 1989: 58), demostrándose una clara homogeneidad en la cultura material inmueble. No existe ningún sitio con edificios únicos o exclusivos que pudiera considerarse la capital o el centro de gobierno. Los 26 sitios que presentan montículos con rampa comparten elementos característicos, sin resaltar ninguno de ellos por diferenciarse de los otros más que en el número de montículos. Estas observaciones, combinadas con el material cultural mueble y los datos etnohistóricos, nos llevan a concluir la idea previamente mencionada y que desarrollaremos a manera de conclusión, en el sentido de que los sitios con montículos con rampa constituyeron centros de poder religioso más que político, organizados entre sí en forma heterárquica.

El análisis de las fuentes etnohistóricas concluye que existieron tres poblaciones principales en el área de estudio: Otavalo, Caranqui y Cayambe. Estos datos no coinciden cabalmente con las informaciones que nos proveen las fuentes arqueológicas. Si bien en estos tres sitios hay evidencias de ocupaciones prehispánicas y las tres muestran restos de arquitectura monumental (Athens 1980), solamente Puntiachil (Cayambe) se considera a partir de las fuentes arqueológicas uno de los sitios principales en función de la presencia de montículos artificiales con rampa. Ni Otavalo ni Caranqui presentan evidencias de este tipo de rasgos arquitectónicos, si bien hay que tomar en cuenta que de los montículos de Otavalo no sabemos a ciencia cierta si estos estuvieron o no provistos con rampas, ya que cuentan entre los conjuntos de montículos con mayor grado de deterioro (Athens 1980: 159). En cuanto a Caranqui, tampoco contamos con evidencias de la existencia de montículos con rampa, y la mayoría de las investigaciones realizadas en este sitio se concentran más bien en los hallazgos de la época incaica (Bray y Echeverría 2014). De otro lado, los sitios con mayor número de montículos artificiales, Zuleta y Cochasquí, no son mencionados en los documentos tempranos como poblaciones principales. Como se indicó en el análisis individual de las fuentes etnohistóricas, el principal indicador al que éstas hacen alusión es la población, es decir que la importancia de estas tres regiones (Otavalo, Caranqui y Cayambe) estaría definida a partir de la cantidad de habitantes con la que contaban. Precisamente en este punto parece radicar el problema de la incompatibilidad de esta información con los datos arqueológicos, y se debe a que se asume que los sitios con montículos fueron centros de poder político, y por tanto debían estar densamente poblados.

Nosotros creemos aquí que las evidencias arqueológicas apuntan a que los sitios monumentales, y en particular aquellos con montículos con rampa, no corresponden -al menos no primordialmente- a estructuras domésticas ni habitacionales, como hemos planteado anteriormente (Ugalde 2015). En el mismo sentido entendemos la interpretación de Wurster, cuando al resumir los resultados de las excavaciones en la pirámide «E» de Cochasquí, recalca que los hornos no necesariamente implican que estas construcciones fueran de carácter doméstico, sino que pudieron ser de carácter ceremonial (Wurster 1989: 62). La cantidad de comida que se pudo preparar en 
ellos habla a favor de una interpretación en ese sentido. Su contexto también, pues las rampas de acceso a la pirámide, que desembocan en el centro de la plataforma y proporcionan paso directamente a las estructuras circulares, hacen pensar en grandes cantidades de personas ascendiendo a ellas y tomando parte en alguna festividad. El largo excesivo de las rampas no es funcional para un efecto estrictamente residencial, y hace pensar más bien en un prolongamiento exagerado y premeditado de la llegada a la plataforma, intencionado para propiciar un ambiente de ritualidad. Está claro que lo que se pretendió con la construcción de estas rampas es extender (innecesariamente desde un punto de vista funcional) el tiempo de caminata requerido para alcanzar la plataforma del montículo. Esto debió concebirse para causar un efecto relacionado con la ceremonialidad de la celebración, que creemos tuvo lugar sobre las plataformas con la participación masiva de la población y la repartición de abundante comida y bebida, especialmente chicha, como ha resaltado Athens tras observar la gran cantidad de fragmentos de ánforas que quedaron en estos montículos (Athens 2010, 2012).

En nuestra interpretación, los montículos con rampa cumplieron con una función cívico-religiosa enmarcada en el sistema de reciprocidad propio de las sociedades complejas de los Andes. Proponemos como hipótesis (a verificarse con mayores investigaciones arqueológicas) que los montículos artificiales que tienen forma de pirámide truncada con plataforma, y sin rampa, pudieron constituir las residencias de la clase alta. En todos los sitios donde se han registrado montículos con rampa, existen también otros sin rampa, generalmente de menor tamaño. Como ejemplo podemos mencionar a los montículos A, D, $\mathrm{H}^{7}$ y $\mathrm{N}$ de Cochasquí (Oberem y Wurster 1989) así como los montículos AA, M, N, Q, X y V de Zuleta (Athens 2010).

Las cavidades o artesas excavadas en la pirámide «E» de Cochasquí proporcionan un indicio adicional sobre la organización de estas celebraciones en vinculación directa con la organización interna de la sociedad caranqui y, a la vez, refuerzan la hipótesis de que las pirámides con rampa no fueron sitios habitacionales. Para expresar esta idea, comenzaremos por citar un pasaje rescatado por Bray de los documentos tempranos, específicamente de la crónica de Bernabé Cobo:

«Duraban mucho estos banquetes, y se bebía largo en ellos hasta emborracharse. Sentábanse a comer a la larga en ringlera, cada parcialidad de por sí, a una parte de la hanansaya, y a otra, la de hurinsaya, en frente una de otra, como dos líneas paralelas, y brindaban los de la una a los de la otra por este orden: el que brindaba a otro se levantaba de su lugar e iba para él con dos vasos de chicha en las manos, y dando al otro el uno, se bebía el otro, bebiendo ambos a la par» (Cobo 1964 [1653]: Libro 14, Cap. 5 , p. 245, citado en Bray 2003: 143).

Esta cita alude, primero, al bien sabido hecho de que en los Andes eran frecuentes las grandes celebraciones en las que tomaban parte todos los integrantes de los $a y$ llus. Hemos de entender aquí que Cobo se refiere con parcialidades a los dos grupos

7 El montículo $\mathrm{H}$ es el único de los montículos sin rampa de Cochasquí que fue excavado por Oberem y su equipo, y que ya había sido intervenido por excavaciones ilegales. Se ubica muy cerca de las pirámides con rampa G, J y K, y la excavación realizada sacó a la luz una superficie de barro cocido así como una cavidad (artesa) muy similar a las registradas en las plataformas de las pirámides con rampa de este mismo sitio (Wurster 1989: 39). 
(podría decirse mitades $^{8}$, aunque no necesariamente tenía que ser idéntico el número de personas en cada uno) que conformaban el ayllu, y que responden al concepto de opuestos complementarios que en la cosmovisión andina se definen como hanan y hurin. De otro lado, y más concretamente, indica una clara organización espacial de estas parcialidades en el marco de las celebraciones, lo cual nos hace pensar que tales reglas estrictas debieron dejar algún tipo de huella en el registro arqueológico. Ahora bien, revisando los hallazgos de la plataforma de la pirámide «E» de Cochasquí, que es la única que ha sido excavada en su totalidad, nos encontramos con lo que podría ser una evidencia material de estas costumbres. En esta plataforma, Wurster (1989) excavó dos planchas de barro cocido de forma circular y con evidencias de techamiento. En cada una de estas planchas se encuentran dos cavidades o artesas que han sido interpretadas como hornos, tanto para Cochasquí como para otros sitios del área cultural caranqui que han reportado este tipo de hallazgos (Athens 1980). En estas cavidades se observan piedras de forma cónica dispuestas en varios conjuntos, formando triángulos. Estos conjuntos han sido interpretados como tulpas, es decir estructuras destinadas a sostener vasijas. A juzgar por el número de tulpas de los hornos de la pirámide «E» de Cochasquí, se preparó allí una considerable cantidad de alimentos simultáneamente. La evidencia directa ${ }^{9}$ nos muestra 13 tulpas, pero probablemente el número fue mayor ya que hay espacios en los que no se encontraron las piedras cilíndricas pero pudieron haber existido anteriormente. En todo caso se cocinó al menos en 13 vasijas a la vez, con lo cual se pudieron preparar sin problemas alimentos para varios cientos de personas. Llama la atención en las planchas de barro cocido de la pirámide «E» de Cochasquí, que ambas reproducen el mismo patrón en la distribución espacial de las cavidades; en ambos casos hay dos cavidades, la de la izquierda mayor en tamaño que la de la derecha. Nos aventuramos, en función del texto de Cobo citado, a postular que cada cavidad correspondía a una parcialidad del ayllu que celebraba la fiesta (que vendría a ser en este caso el ayllu o uno de los ayllus de Cochasquí), en las que se preparaba por separado la comida que luego se ofrecía bajo el sistema de reciprocidad referido por Cobo. Es decir, que las plataformas de las pirámides contendrían una evidencia material del tipo de organización dual del interior de las comunidades caranqui, reflejada claramente en el espacio físico. Estas celebraciones constituyeron en los Andes el principal elemento cohesionador en el interior de las sociedades, y se usaron con frecuencia como herramienta eficaz para mantener el statu quo y desestimar las desigualdades sociales.

Asumiendo entonces que los complejos de montículos fueron espacios ceremoniales más que residenciales, esto explicaría por qué estos sitios no son mencionados como grandes centros poblados en los documentos escritos de la época colonial temprana. De los pocos complejos monumentales en los que se ha excavado también en las áreas planas (es decir fuera de los montículos), en Cochasquí se han encontrado puntualmente restos habitacionales en el sector oriental de las pirámides (Ugalde

8 En este sentido, es más apropiado el término utilizado en inglés de moiety, que significa mitad, pero también fracción o porción, y que algunos autores por este motivo prefieren utilizar.

9 Ver dibujos y fotografías de estos hallazgos en Oberem y Wurster 1989, así como la descripción detallada e interpretación de los mismos en Wurster 1989. 
2015; Wentscher 1989), mientras que en Socapamba y Otavalo las investigaciones de Athens sacaron a la luz una mínima cantidad de artefactos en los espacios intermonticulares (Athens 1980: 166, 175). Ya se ha propuesto para otras regiones una interpretación heterárquica para sociedades con arquitectura monumental desde criterios similares (Dillehay 2006).

Entendidos en este sentido, los montículos artificiales con rampa pudieron ser la representación material de una ideología caranqui que a su vez pudo haber sido el eje de la articulación social a nivel regional y el argumento legitimador del poder y el control en el interior de los grupos constituyentes del conjunto, pero que entre ellos funcionaban de forma autónoma.

AgRadecimientos: La realización de esta investigación fue posible gracias al auspicio del Gobierno de la Provincia de Pichincha y al apoyo institucional de la Pontificia Universidad Católica del Ecuador y el Instituto Nacional de Patrimonio Cultural. Colaboraron en la revisión y fichaje de informes de arqueología de rescate los estudiantes Tamia Viteri, Daniel Soria y Kyra Torres.

\section{Referencias bibliográficas}

Ango de Salazar, Lorenzo

1648 Petición de don Lorenzo Ango de Salazar gobernador y cacique en su nombre y el de otros caciques. Otavalo: AN/Q, Indígenas No.5, $5 \mathrm{fls}$.

ANÓNIMO

1992 «La ciudad de San Francisco de Quito» [1573], en Relaciones histórico-geográficas de la Audiencia de Quito, siglo XVI-XIX, Pilar Ponce, ed., pp.187-222. Quito: Abya-Yala, Marka Instituto de Historia y Antropología Andina.

ATHENS, John Stephen

1980 El proceso evolutivo en las sociedades complejas y la ocupación del periodo Tardío-Cara en los Andes Septentrionales del Ecuador. Otavalo: Instituto Otavaleño de Antropología.

2003 Inventory of Earthen Mound Sites, Northern Highland Ecuador. Informe inédito. Quito: Instituto Nacional de Patrimonio Cultural.

2010 El sitio Tola de la Hacienda Zuleta: investigaciones geofisicas 2005 y 2009. Informe inédito. Quito: Instituto Nacional de Patrimonio Cultural.

2012 El sitio Tola de la Hacienda Zuleta: Investigaciones 2010. Informe inédito. Quito: Instituto Nacional de Patrimonio Cultural.

Betanzos, Juan de

1987 Suma y narración de los incas [1553], tomo 5. Madrid: Biblioteca Hispano-Ultramarina.

BorJa, Antonio

1992 «Relación en suma de la doctrina e beneficio de Pimampiro y de las cosas notables que en ella hay, de las cuales es beneficiado el P. Antonio Borja» [1581], en Relaciones Geográficas de Indias. Perú, Marcos Jiménez de la Espada, ed., tomo 3, pp. 248-253. Biblioteca de Autores Españoles, 184. Madrid: Atlas. 
BRAY, Tamara L.

1995 «The Panzaleo Puzzle: Non-local Pottery in the Northern Highland Ecuador». Journal of Field Archaeology 22 (2): 137-156.

2001 Vínculos andinos amazónicos en la prehistoria ecuatoriana: el distrito Pimampiro y el sitio Shanshipamba. Fase III: excavaciones en el sitio de Shanshipamba. Informe inédito. Michigan.

2003 Los efectos del imperialismo incaico en la Frontera Norte. Quito: Abya-Yala, Marka Instituto de Historia y Antropología Andina.

2008 «Late Pre-Hispanic Chiefdoms of Highland Ecuador», en Handbook of South American Archaeology, Helaine Silvermann y William H. Isbell, eds., pp. 527543. Nueva York: Springer.

BRAY, Tamara L. y José ECHEVERRÍA

2014 «Al final del Imperio: el sitio arqueológico Inca-Caranqui en la Sierra Septentrional del Ecuador». Antropología. Cuadernos de Investigación 13: 127-150.

BRITO, Christian, Jonathan DAMP y Esteban AcosTA

2014 «La arqueología de San Pablo y Otavalo: centros de poder y la teoría de los lugares centrales», en Estudios multidisciplinarios en cinco espacios prehispánicos tardios del Ecuador, pp. 136-150. Quito: Secretaría Nacional de ESCTI, Instituto Nacional de Patrimonio Cultural, Universidad de Cuenca.

Buys, Josef, Oscar Manosalvas y Byron Camino

1991 Prospección arqueológica en Cayambe. Informe inédito. Quito: Instituto Nacional de Patrimonio Cultural.

CAillavet, Chantal

2000 Etnias del norte. Etnohistoria e historia de Ecuador. Quito: Instituto Francés de Estudios Andinos, Abya-Yala.

Cordero, María Auxiliadora

2009 El Cacicazgo Cayambi. Trayectoria hacia la complejidad social en los Andes Septentrionales. Quito: Abya-Yala.

Crumley, Carole L.

1979 «Three Locational Models: An Epistemological Assessment for Anthropology and Archaeology». Advances in Archaeological Method and Theory 2: 141-173.

1995 «Heterarchy and the Analysis of Complex Societies», en «Heterarchy and the Analysis of Complex Societies», Robert Ehrenreich, Carole Crumley y Janet Levy, eds. Archeological Papers of the American Anthropological Association 6 (1): 1-5.

CURrIE, Elisabeth J.

2001 «A Late Period Caranqui Chiefdom in the Northern Highlands of Ecuador: Archaeological Investigations at Hacienda Zuleta». Internet Archaeology 10. http:// intarch.ac.uk/journal/issue10/currie_index.html

DillehaY, Tom

2006 «Organización y espacios sociopúblicos incipientes: tres casos de los Andes». Boletín de Arqueología PUCP 10: 13-36.

DYRDAHL, Eric

2015 «Obtención de obsidiana en un sector no monumental de Cochasquí», en Cochasqui revisitado. Historiografía, investigaciones recientes y perspectivas, $\mathrm{M}^{\mathrm{a}} \mathrm{Fer}-$ nanda Ugalde, pp. 123-132. Quito: Gobierno Autónomo Provincial de Pichincha. 
Dyrdahl, Eric y Robert J. SPEAKMAN

2013 «Investigating Obsidian Procurement at Integration Period (ca. AD 700-1500): Tola Sites in Highland Northern Ecuador via Portable X-Ray Fluorescence (pXRF)». Archaeological Chemistry 8: 211-232.

Ehrenreich, Robert M., Carole L. Crumley y Janet E. Levy (eds.)

1995 «Heterarchy and the Analysis of Complex Societies». Archeological Papers of the American Anthropological Association 6 (1).

FrITZ, Uwe y Uwe SCHÖNFELDER

1986 «New Results Concerning the Integration Period in the Northern Highlands of Ecuador». Bulletin of the Institute of Archaeology 23: 127-150.

GONDARD, Pierre y Freddy LóPEz

1983 Inventario arqueológico preliminar de los Andes Septentrionales. Quito: Instituto Geográfico Militar.

Hageman, Jon B. y Jon C. Lohse

2003 «Heterarchy, Corporate Groups, and Late Classic Resource Management in Northwestern Belize», en Heterarchy, Political Economy, and the Ancient Maya: The Three Rivers Region of the East-Central Yucatán Peninsula, Vernon Scarborough, Fred Valdez Jr. y Nicholas Dunning, eds., pp. 109-121. Tucson: University of Arizona Press.

JIJÓN Y CAAMAÑo, Jacinto

1997 Antropología prehispánica del Ecuador [1952]. Quito: Museo Jacinto Jijón y Caamaño, Embajada de España, Agencia Española de Cooperación Internacional y Editorial Santillana.

LANDÁzURI, Cristóbal

1995 Los curacazgos pastos prehispánicos: agricultura y comercio, siglo XVI. Quito: Banco Central del Ecuador, Abya Yala, Instituto Otavaleño de Antropología.

LARRAÍn BARROS, Horacio

1980 Demografía y asentamientos indígenas en la Sierra Norte del Ecuador en el siglo XVI. Otavalo: Instituto Otavaleño de Antropología.

MegGers, Betty

1966 Ecuador. Ancient Peoples and Places. Londres: Thames \& Hudson.

MEYERS, Albert

1981 «Análisis de la cerámica de Cochasquí», en Cochasquí: estudios arqueológicos, Udo Oberem, ed., pp. 219-268. Otavalo: Instituto Otavaleño de Antropología.

1989 «Análisis de la cerámica tosca», en Excavaciones en Cochasquí, Ecuador 19641965, Udo Oberem y Wolfgang Wurster, eds., pp. 180-197. Mainz am Rhein: Verlag Philipp von Zabern.

Moreno YÁnez, Segundo

1982 «Formaciones políticas tribales y señoríos étnicos», en Nueva Historia del Ecuahdor, vol. 2, Enrique Ayala, ed., pp. 9-134. Quito: Corporación Editora Nacional, Grijalbo.

2007 Historia antigua del País Imbaya. Quito: Universidad de Otavalo.

Morris, Craig y Donald E. THOMPSON

1970 «Huanuco Viejo: An Inca Administrative Center». American Antiquity 35 (3): 344-362. 
Moseley, Michael E.

1992 The Incas and their Ancestors. The Archaeology of Peru. Londres: Thames \& Hudson.

MURRA, John V.

1975 «El control vertical de un máximo de pisos ecológicos en la economía de las sociedades Andinas» [1972], en Formaciones económicas y políticas del mundo andino, pp. 59-115. Lima: Instituto de Estudios Peruanos.

NARR, Karl y Uwe ScHÖNFELDER

1989 «Fechas de carbono 14», en Excavaciones en Cochasquí, Ecuador 1964-1965, Udo Oberem y Wolfgang Wurster, eds., pp. 177-179. Mainz am Rhein: Verlag Philipp von Zabern.

OBEREM, Udo

1981 «El acceso a recursos naturales de diferentes ecologías en la Sierra ecuatoriana (siglo XVI)», en Contribución a la etnohistoria ecuatoriana, Segundo Moreno y Udo Oberem, eds., pp. 45-71. Otavalo: Banco Central del Ecuador, Abya Yala, Instituto Otavaleño de Antropología.

Oberem, Udo (ed.)

1981 Cochasquí: estudios arqueológicos. Otavalo: Instituto Otavaleño de Antropología.

Oberem, Udo, Wolfgang W. Wurster, Roswith Hartmann y Jürgen Wentscher

1968 «Die Bergfestung Quitoloma im nördlichen Hochland Ecuadors». Baessler Archiv 16: 331-356.

OBEREM, Udo y Wolfgang Wurster (eds.)

1989 Excavaciones en Cochasquí, Ecuador 1964-1965. Mainz am Rhein: Verlag Philipp von Zabern.

Paz Ponce de León, Sancho

1992 «Relación y descripción de los pueblos del partido de Otavalo» [1582], en Relaciones histórico-geográficas de la Audiencia de Quito, siglo XVI-XIX, Pilar Ponce, ed., pp. 359-371. Quito: Marka Instituto de Historia y Antropología Andina, AbyaYala.

Plaza Schuller, Fernando

1977 El complejo de fortalezas de Pambamarca. Contribución al estudio de la arquitectura militar prehispánica en la Sierra Norte del Ecuador; Segundo Informe Preliminar. Otavalo: Instituto Otavaleño de Antropología.

PotTER, Daniel R. y Eleanor M. KING

1995 «A Heterarchical Approach to Lowland Maya Socioeconomies», en «Heterarchy and the Analysis of Complex Societies», Robert Ehrenreich, Carole Crumley y Jane Levy, eds. Archeological Papers of the American Anthropological Association 6 (1): 17-32.

PuENTO, Hierónimo

1974 «Probanza de servicios de Hierónimo Puento Cacique principal del pueblo de Cayambe» [1583], en Documentos para la historia militar, tomo I, pp. 13-50. Quito: Dirección de Historia y Geografía Militar del E.M.C. de las FF.AA., Casa de la Cultura. 
$M^{a}$ Fernanda Ugalde y Cristóbal Landázuri Sociedades heterárquicas en el Ecuador prehispánico...

RAMón VALAREZO, Galo

1987 La resistencia andina: Cayambe 1500-1800. Quito: Centro Andino de Acción Popular.

RosTwOrOwsKy, María

1988 Historia del Tahuantinsuyu. Lima: Instituto de Estudios Peruanos.

SCHÖNFELDER, Uwe

1989 «Análisis de la cerámica fina del tipo Panzaleo-Cosanga y hallazgos menores», en Excavaciones en Cochasquí, Ecuador 1964-1965, Udo Oberem y Wolfgang Wurster, eds., pp. 198-217. Mainz am Rhein: Verlag Philipp von Zabern.

Ugalde, María Fernanda

2007 «Formas de enterramiento en Quito preincaico: Los descubrimientos de Ciudad Metrópoli». Antropología. Cuadernos de Investigación 7: 6-33.

2015 Cochasqui revisitado. Historiografia, investigaciones recientes y perspectivas. Quito: Gobierno de la Provincia de Pichincha.

Uhle, Max

1933 «Die Ruinen von Cochasquí (nördlich von Quito)». Ibero-Amerikanisches Archiv 7 (2): $127-134$

1939 «Las ruinas de Cochasquí». Boletín de la Academia Nacional de Historia 18 (54): $5-14$.

VARGaS, José María

1977 Historia del Ecuador, siglo XVI. Quito: Pontificia Universidad Católica de Ecuador.

VILLALBA, Marcelo

2009 Prospección arqueológica, Proyecto Hidroeléctrico Chespi, Provincia de Pichincha. Informe inédito. Quito: Instituto Nacional de Patrimonio Cultural.

WENTSCHER, Jürgen

1989 «Montículos y otras áreas de excavación», en Excavaciones en Cochasquí, Ecuador 1964-1965, Udo Oberem y Wolfgang Wurster, eds., pp. 70-97. Mainz am Rhein: Verlag Philipp von Zabern.

WURSTER, Wolfgang

1989 «Ruinas existentes», en Excavaciones en Cochasquí, Ecuador 1964-1965, Udo Oberem y Wolfgang Wurster, eds., pp. 11-69. Mainz am Rhein: VerlagPhilipp von Zabern.

Zalles-Flossbach, Carlos

1989 Los artefactos líticos de Cochasquí, Ecuador. Tesis de Maestría. Bonn: Rheinische Friedrich-Wilhelms-Universität. 